\title{
HESPER, EL VESPROY EL VESPERTILIO: ELEMENTOS DE CONTINUIDAD ENTRE EL MILENARISMO STAÚFICO $Y$ EL CICLO PROFÉTICO DEL IMPERIO ARAGONÉS
}

\author{
Manuel Alejandro RODRÍGUEZ DE LA PEÑA \\ Universidad Autónoma de Madrid
}

El fenómeno de la profecía política alcanzó a lo largo del siglo XIII una relevancia sin precedentes en el marco de la controversia teórica entre los publicistas defensores de las diferentes posiciones de los poderes políticos de la época'. La publicística del siglo XIII desarrolló sus controversias fundamentalmente en dos niveles: 1/ el enfrentamiento entre las posiciones hierocráticas del Sacerdotium y las soberanistas del Regnum y 2/ la disputa por el dominium Mundi entre las Monarquías de Occidente, disputa íntimamente ligada al surgimiento de una conciencia protonacional en el seno de éstas.

Aunque el ciclo profético del Imperio aragonés está inscrito en este segundo nivel, las peculiares circunstancias en que tuvo lugar su alumbramiento le confirieron un marcado perfil gibelino más propio del escenario de enfrentamiento entre Hierocracia y Monarquía, perfil que únicamente tiene explicación a la luz de la herencia política staúfica ímplicita en el nacimiento de la vocación imperial del Casal d'Aragó.

1 A este respecto, la figura de Joaquín de Fiore (1135-1202) como prestigiadora del profetismo escatológico marca un hito en la influencia política del milenarismo. Aunque hay precedentes anteriores de utilización política de la profecía (son los casos de Raúl GLABER y su De divina Quaternitate, Godofredo de MONMOUTH y la Prophetia Merlini o el del ciclo escatológico de la Primera Cruzada estudiado por Paul ALPHANDÉRY en La Chretienté et l'idee de Croisade, 1954, pp. 160-208) no sería hasta el siglo XIII cuando se produjera su eclosión definitiva como uno de los factores formadores de ideología política (cf. Hans M. SCHALLER, «Endzeit-Erwartung und Antichrist-Vorstellungen in der Politik des 13. Jahrhunderts", Festschrift für Hermann Heimpel, Gotinga, 1971, p. 924). 
Por ello, es preciso proceder a una delimitación de aquellos elementos del ciclo profético catalán que, procedentes de la mística imperial de la Casa de Suabia, arrojen luz sobre la particular idiosincrasia del programa político universalista de los reyes que sucedieron a Jaime I en el trono aragonés.

La principal dificultad de esta tarea radica en la díficil diferenciación entre elementos pertenecientes a la tradición milenarista propiamente imperial y elementos joaquinitas, pese al claro carácter hierocrático de estos últimos.

La razón de esta dificultad estriba en el hecho de que ambos ciclos proféticos compartieron un mismo lenguaje simbólico y una misma herencia escatológica de raigambre bíblica, difiriendo tan sólo en la interpretación última de los hechos anunciados. Es esta interpretación y la significación política que le es otorgada, la que normalmente delatará las tendencias güelfas o gibelinas del que formula las profecías ${ }^{2}$.

Sin embargo, como veremos más adelante, es posible identificar algunas figuras alegóricas propias del ciclo escatológico de los Staufen, figuras que, quizá debido a su origen pagano, no aparecen en la tradición joaquinita. Estas figuras alegóricas son la expresión plástica de algunos arquetipos fundamentales de la ideología imperial staúfica y su recepción en el corpus de profecías catalanas resulta altamente significativo a la hora de establecer posibles líneas de continuidad entre el proyecto imperial de la regia stirps de Waiblingen y el de la dinastía catalano-aragonesa.

\section{CARACTERIZACIÓN DE LA INFLUENCIA JOAQUINITA EN EL CICLO PROFÉ- TICO CATALANO-ARAGONÉS}

Marjorie Reeves ha establecido que la evidencia de influencia joaquinita en un texto de profecía política medieval no consiste en atestiguar la creencia del autor en el carácter profético de Joaquín de Fiore o su adhesión a la teoría de las Tres Edades del Mundo, sino más bien en una determinada visión de la historia basada en la expectación milenarista ante una inminente Era de iluminación y beatitud, un auténtico tempus gratiae previo a la venida de Cristo $^{3}$.

De esta forma, podemos' hablar perfectamente de influencia joaquinita en un gran número de autores de los siglos XIII y XIV sin por ello hacer de ellos adeptos de las doctrinas trinitarias del Evangelio Eterno ${ }^{4}$. En este contexto debemos enmarcar la penetración en Aragón del joaquinismo.

Alain Milhou ha señalado como principales introductores de los ciclos proféticos

2 En particular esto es válido para las pseudo-profecías realizadas ex eventu, es decir, aquellas acerca de hechos ya ocurridos sobre los que el profeta proyecta una predicción que los reinterpreta y sitúa en un marco espacio-temporal más amplio.

3 Cf. Marjorie REEVES, «The originality and influence of Joachim of Fiore», Traditio, XXXVI, 1980, p. 298; es esta creencia la que diferencia el joaquinismo de otros movimientos milenaristas anteriores, pues éstos situaban siempre la Edad de la perfección en un tiempo posterior al Juicio Final.

4 Este sería el caso, entre otros, de San Buenaventura, Roger Bacon, Juan de París, Dante o Gerhold de Reichenberg (M. REEVES, op. cit., pp. 299-304). 
milenaristas en España a los franciscanos catalanes imprégnes de joachimisme ${ }^{5}$. En esta dirección, sin duda fue Arnaldo de Vilanova, hombre cercano a la espiritualidad beguina, la figura que primero recogió el pensamiento del abad de Calabria en Aragón, siendo su influencia decisiva para la posterior expansión de las ideas joaquinitas al sur de los Pirineos ${ }^{6}$. Esta influencia es atestiguada por los cánones del concilio de Tarragona de 1317, que revelan la preocupación que despertaba en la jerarquía eclesiástica el gran número de sus seguidores fraticelos y beguinos ${ }^{7}$.

Las concepciones filosóficas del sabio catalán se apoyaban en la formación de un homo novus que alcanzaría la perfección física (gracias a la medicina y la alquimia, que alcanzan en su pensamiento una dimensión escatológica) y espiritual en la Tertia Aetas anunciada por Joaquín de Fiore $^{8}$.

En este sentido, no es un hecho casual el que las meditaciones milenaristas de Arnaldo de Vilanova comenzaran con un comentario al De semine scripturarum de Joaquín de Fiore $^{9}$. Pronto le seguiría la Expositio super Apocalypsi ${ }^{10}$, fuertemente imbuida de las tesis trinitarias joaquinitas. Ambas obras fueron presentadas por su autor ante el papa Clemente $V$ en Burdeos.

Estas obras proféticas del visionario catalán no se limitaron a reproducir las pautas hierocráticas de las predicciones de Joaquín de Fiore ${ }^{11}$, sino que las pusieron al servicio de los designios seculares del Casal d'Aragó.

No en vano, Arnaldo de Vilanova era ante todo un hombre estrechamente ligado a la Corona catalano-aragonesa. Había sido el médico personal de Pedro III y en el momento de la redacción de estas dos obras (1297-1302) era consiliarium et familiarem del Rey Jaime $\mathrm{I}^{12}$, para el que había desempeñado labores diplomáticas en la corte de Francia. Por ello, no es aventurado afirmar que se erigía en portavoz de los más

5 Cf. Alain MILHOU, «La Chauve-Soris, le Nouveau David et le Roi Cache (trois images de l'empereur des dernier temps dans le monde ibérique)", Melanges de la Casa de Velazquez, XVIII, 1982 , p. 62.

6 Cf. M. REEVES, The influence of Prophecy in the Later Middle Ages: a study in Joachimism, Oxford, 1969, p. 221: In Aragon and Catalonia the chief carriers of Joachimist ideas may well have been the works of Arnold of Villanova...

7 Cf. José María POU Y MARTÍ, Visionarios, beguinos y fraticelos catalanes (siglos XIII-XIV), Vich, 1930, p. 100; 198-220.

8 Cf. MÉNENDEZ PELAYO, Historia de los Heterodoxos españoles, II, p. 479, Madrid, 1986 (4ª ed.), y Morton W. BLOOMFIELD, "Joachim of Flora: a critical survey on his canon, teachings, sources, biography and influence», Traditio, XIII, 1957, p. 301.

9 Códice 3824 de la Biblioteca Vaticana (cf. Menéndez Pelayo, op. cit., p. 491).

10 Códice 5740 de la Biblioteca Vaticana (cf. Menéndez Pelayo, op. cit, p. 492).

11 Robert E. Lerner opina que Joaquín de Fiore fue ante todo un propagandista de la Hierocracia; la figura central de su ciclo profético era un Papa angélico que debería ser simul Regem et sacerdotem secundum ordinem Melchisedech (Concordia Novi et Veteri Testamenti, IV); era la versión escatológica de una imagen que acuñó en lo político Inocencio III (cf. "Joachim of Fiore as a link between St. Bernard and Innocent III on the figural significance of Melchisedech", Mediaeval Studies, 42,1980 ). Reeves (cf. op. cit., p. 63) señala que el favor mostrado por los papas hacia el abad fue strikingly consistent.

12 A.C.A., Reg. $n^{\circ}$ 193, fol. 69v; Lérida, Abril de 1302. 
íntimos anhelos de su soberano cuando proclamaba que la dinastía aragonesa estaba llamada a la Monarchia Mundi.

En efecto, el comentario de Vilanova a la profecía joaquinita Vae Mundo in centum annis anunciaba ${ }^{13}$ que surgiría de España un Rey que destruiría la machometicae pravitas y que, tras someter África y Egipto, alcanzaría la Monarquia ${ }^{14}$ del orbe.

Pero no fue esta la principal innovación introducida por el visionario catalán en el esquema joaquinita. De hecho, con ello no hacía sino aplicar a España el viejo arquetipo milenarista del emperador de los últimos tiempos, arquetipo que ya había sido incorporado a lo largo del siglo XIII a los ciclos proféticos joaquinitas (aunque no estaba presente en la obra original del abad de Fiore, que únicamente contemplaba la figura del Papa angélico ${ }^{15}$ ).

La importancia de la novedad introducida por la profecía vilanoviana radica en su utilización de una imagen alegórica cargada de significado: la figura del Vespertilio. Esta figura, cuyas implicaciones serán analizadas en profundidad más adelante, era la imagen del Murciélago que Jaime I había dado a Valencia y Mallorca como emblema, el Rat penat que simbolizaba de algún modo al Casal d'Aragó en la mentalidad popular ${ }^{16}$.

Sin embargo, el emblema de Jaime el Conquistador no era un Murciélago sino en la imaginación popular ${ }^{17}$. Se trataba en realidad de un Drac alat, el Dragón de San Jorge ${ }^{18}$, que Arnaldo de Vilanova asimiló al Dragón Alado del Apocalipsis ${ }^{19}$. Esta elección simbólica no fue casual pues enlazaba directamente con la tradición escatológica staúfica y, lo que es aún más importante, situaba claramente a la profecía en el ámbito del ciclo profético imperial más opuesto al mensaje hierocrático joaquinita ${ }^{20}$.

13 Hyspania, nutrix machometicae pravitatis, reciproca furia laniabitur nam in invicem ipsius regna consurgent... subjiciensque Africam et Caput Bestiae conterens accipiet Monarchiam et in posteris humiliet Nili habitatores (apud Pou Martí, op. cit., p. 55).

14 A la luz de la teoría política medieval hay que interpretar la frase de Vilanova accipiet Monarchiam en el sentido de una aspiración al Imperio universal. El concepto Monarchia se reservaba en el lenguaje medieval exclusivamente para designar a los estados mundiales (aunque lo fueran sólo conceptualmente, como en el caso del Imperio Romano Germánico), mientras que el resto de las formaciones políticas recibían el nombre de Regnum.

15 La figura del emperador de los últimos tiempos era propia del ciclo escatológico imperial bizantino, siendo aplicada repetidamente a la Casa de Suabia por sus partidarios a través de la idea de un Tercer Federico. Los joaquinitas respondieron recuperando la idea de un Segundo Carlomagno (que sería francés y no alemán) anunciado como el fiel ejecutor de los designios del Papa Angélico, de la misma forma que la Francia de los Capetos actuaba en esos años como la obediente hija de la Iglesia. Hasta que se produjo esta recepción la figura del emperador de los últimos tiempos representaba para los joaquinitas una alegoría del Anticristo (cf. Marjorie REEVES, «Joachimist influences on the idea of a Last World Emperor», Traditio, XVII, 1961, p. 328).

16 Cf. A. MILHOU, art. cit., pp. 63-69.

17 No sería hasta el siglo XVII el que la transformación del Dragón en Murciélago se hiciera oficial en la heráldica (cf. A. MILHOU, loc. cit.).

18 Proclamado solemnemente en 1344 como emblema de la Corona de Aragón.

19 Cf. A. MILHOU, loc. cit., y J.M. POU MARTÍ, op. cit., pp. 56-59.

20 R. Manselli cuestiona abiertamente que Vilanova estuviera influenciado por el joaquinismo (cf. La Religiosità d'Arnaldo de Villanova, Roma, 1951, pp. 20-23). 
En el marco de una estricta simbología profética la elección vilanoviana del Dragón alado como símbolo era de una enorme trascendencia. De hecho, la sexta y la séptima capita Draconis del Apocalipsis habían sido interpretadas por Joaquín de Fiore y los joaquinitas como símbolos respectivos del anunciador del Anticristo y del propio Antichristus pessimus ${ }^{21}$.

Teniendo en cuenta que era opinión comúnmente aceptada entre los joaquinitas que Federico II había sido la sexta cabeza del Dragón $n^{22}$ y que de su sangre nacería el Anticristo (asimilado al Tercer Federico esperado por los gibelinos) ${ }^{23}$, se puede calibrar el trasfondo de la imagen utilizada por Vilanova quien era, no lo olvidemos, amigo y consejero del Rey Federico III de Sicilia ${ }^{24}$, un Staufen por linea materna muy mal visto en la Curia.

La elección de la imagen del Dragón por Vilanova es indicativa de una consciente opción gibelina poco conciliable con la simbología joaquinita. La imagen vilanoviana sólo cobra pleno sentido a la luz de su obra profética fundamental, el $D e$ adventu Antichristi et fine Mund ${ }^{25}$ (año 1299), donde se desata en invectivas contra el clero, clama por la reforma de la Iglesia y anuncia la venida del Anticristo para 1345 . Esta faceta destructora, purificadora y, en último término, reformadora, es la que encarnaba el Dragón apocalíptico en el pensamiento escatológico gibelino.

En los ciclos proféticos imperiales el Tercer Federico siempre asumía una función destructora, de reforma violenta y traumática de la Iglesia. Así, el hereje dominico fray Arnoldo de Suabia proclamaba en su De correctione Ecclesiae (año 1248) la venida de un emperador de los últimos tiempos que reformaría a sangre y fuego la depravada Iglesia romana dentro de una necesaria Sexta Era de tribulación ${ }^{26}$.

De un modo similar, el mensaje vilanoviano contenido en el De adventu Antichristi era tan subversivamente anti-hierocrático que los teólogos de la Universidad de París consiguieron hacerle encarcelar (pese a su condición de embajador de Aragón) orde-

21 Cf. M. REEVES, «The idea of a Last World Emperor", art. cit., p. 324.

22 Cf. Ernst H. KANTOROWICz, Kaiser Friedrich der Zweite, Munich, 1927, c. I: Joaquín de Fiore anunció a los pocos días del nacimiento de Federico Il que la emperatriz Constanza acababa de dar a luz al tirano universal que anegaria el mundo en un océano de sangre tras regir las naciones con vara de hierro. En las obras pseudo-joaquinitas Super Hieremiam (publ. en Venecia, 1516, fol. 11v) y Praemissiones (ms. Vat. lat. 4959, fol. 2v) se repite esta identificación con la sexta cabeza del Dragón, así como en las Distinctiones de Tomás de Pavía (año 1260, publ. Archivum Franciscanum historicum, XVI, 1923, pp. 27-28).

23 Así lo afirma el tratado pseudo-joaquinita Vaticinium Sibillae Erithreae (año 1252, ed. HolderEgger, en Neues Archiv der Gesellschaft für altere deutsche Geschichtskunde, XV, 1889, p. 168); cf. M. REEVES, art. cit., p. 326, n. 8.

24 P. DIEPGEN, Arnold von Vilanova als Politiker und Laientheologe, Berlín, 1909.

25 Códice 3824 de la Biblioteca Vaticana.

26 Cf. Crónica de Alberto de Stade, M. G. H., Scriptores, XVI, p. 371; M. REEVES, art. cit., p. 326, n. 9; Por otro lado, Robert Graves ha señalado que el Murciélago del escudo de Mallorca está tomado del Khufaash de los sufíes andalusíes cuyo significado es de ruina y destrucción (vid. su introducción a The Sufis de Idries Shah, 1964). 
nando posteriormente el papa Bonifacio VIII que se quemara la obra y se le encarcelara de nuevo en Aviñón ${ }^{27}$.

También de influencia joaquinita son dos anónimos proféticos catalanes de mediados del siglo XIV: los Versos profètics y la Summula super concordia Novi et Veteri Testamentip. Ambos muestran una concepción histórica inspirada en el Evangelio Eterno que incluye la predicción de un glorioso status Mundi tertius, pero difieren, sin embargo, en su inclinación política.

Así, mientras que el autor de los Versos profètics enmarca su visión en el esquema tradicional de Joaquín de Fiore, anunciando las calamidades que sobrevendrán a la Iglesia por obra de un pseudo-profeta elevado al solio pontificio ${ }^{29}$, la Summula, por el contrario, es una obra al servicio de la exaltación mesiánica del Casal d'Aragó.

El desconocido franciscano catalán que compiló la Summula hacia $1368^{30}$ utiliza hábilmente la estructura discursiva del Liber Concordie joaquinita de extrapolación al presente de los hitos temporales del Antiguo Testamento ${ }^{31}$ para insertar en su interior una predicción ex eventu inspirada en la famosa profecía bíblica de las Cuatro Monarquías del Mundo de Daniel (cap. XI).

A pesar de que Reeves califica al autor de la Summula como un perfecto discípulo de Joaquín de Fiore ${ }^{32}$, 10 cierto es que la interpolación introducida por el fraile en la obra joaquinita sigue decididamente la estela de Vilanova.

De otro modo no se puede interpretar la comparación alegórica que realiza entre Alejandro Magno y Pedro III el Grande, comparación por la cual el papel desempeñado por la Macedonia imperial (designada por el profeta Daniel como el macho cabrío) sería ocupado en el siglo XIII por Cataluña ${ }^{33}$, quedando relegados Sicilia y Carlos de Anjou a la triste posición de Persia y el Rey Darío, quienes son vencidos poc en persona por el Rey de Aragón en semblanza de las victorias obtenidas por Alejandro ${ }^{34}$.

27 Cf. MENÉNDEZ PELAYO, op. cit., p. 497.

28 Ambos contenidos en el ms. de la B. N. de Madrid no 6972 (cf. Pere BOHIGAS, «Profecies catalanes dels segles XIV i XV: assaig bibliogràfic", Butlletí de la Biblioteca de Catalunya, VI, 1920-22, pp. 6-7; REEVEs, The influence of Prophecy, op. cit., p. 223.

29 Pseudo propheta culmine Sedis pontificatur, regnabit ficto lumine in seculo sublimatur, clerus perdet Imperium reptans expoliatus ( $v$ v. 24-26, apud Pere Bohigas, loc. cit.). Las únicas concesiones realizadas por el autor a su patria son una apelación a la Virtus yspanis (v. 23) y la sustitución del emperador de los últimos tiempos por los barbari pagani como figura maléfica, único punto en que se aleja del modelo joaquinita.

30 Fecha avanzada por M. REEVES, loc. cit., p. 223.

31 Para Reeves el autor de la Summula es the clearest evidence of a Spanish Joachite who was searching the writings of Joachim at this time and adapting them to contemporary needs (loc. cit., $p$. 223). No otra cosa hizo Arnaldo de Vilanova.

32 ... we see him as a full disciple of Joachim; op. cit., p. 224.

33 ... sicut Persia tangit Ciciliam ita Macedonia Cataloniam (fol. 37, apud. P. Bohigas, art. cit., p. 8). Muntaner avanza esta idea cuando afirma que el Rey Pedro III és lo pus alt hom de cor que anc nasqués d'Alexandri e ençá (cf. Crònica, c. LXXII).

34 Así lo confirma la traducción catalana de la Summula, llamada Refundició compendiada (ms. no 336 de la B. Mun. de Carpentras, apud. P. Bohigas, art. cit., p. 9). 
Pero las visiones imperiales del franciscano catalán no se quedaban ahí. También anunciaba el sometimiento de los reinos hispánicos a la hegemonía de la Casa de Aragón, de la misma forma que Grecia lo había estado a la de Macedonia ${ }^{35}$. Estas profecías realizadas ex eventu, casi un siglo después del Vespro, pueden ser un síntoma de la voluntad de construir en el siglo XIV una plataforma escatológica legitimadora de la conciencia imperial catalana.

Ahora bien, no todo el ciclo profético catalano-aragonés siguió el camino de exaltación monárquica que había iniciado Arnaldo de Vilanova. Hubo obras que se mantuvieron dentro de los márgenes del joaquinismo más estricto. Algunas, incluso, se sumaron con entusiasmo a la publicística más furiosamente güelfa para denunciar al Casal d'Aragó como la estirpe del Anticristo, siendo así fieles continuadoras del ideario hierocrático del abad.

Dentro de la más pura ortodoxia joaquinita ${ }^{36}$ destacan tres obras del ciclo catalán: el anónimo De Statibus Ecclesiae secundum Apocalypsim ${ }^{37}$ (c. 1318), el De Triplici Statu Mundi de Francisco de Eiximenis $(1398)^{38}$ y, sobre todo, las obras del franciscano provenzal Juan de Rocatallada.

La faceta profética del místico franciscano Francisco de Eiximenis plantea, no obstante, algunos problemas de filiación ideológica. Sin lugar a dudas la impronta joaquinita en su obra es evidente, en particular la influencia de Ubertino de Casale ${ }^{39}$, reflejada en la aparición de lugares comunes de los espirituales como las Tres Edades del Mundo y la Reformatio Ecclesiae.

Sin embargo, se aprecian algunos puntos de díficil explicación. El hecho de que fray Eiximenis, pese a su cercanía a la dinastía aragonesa (que había ejercido un continuado mecenazgo sobre su carrera ${ }^{40}$ ), fuera acusado en 1391 por el Rey de predecir para el año 1400 el fin de todas las Monarquías de la Cristiandad a excepción de un triunfante Reino de Francia es cuanto menos desconcertante ${ }^{41}$.

Esta profecía típicamente güelfa, habitualmente incluida en los anuncios de la venida del Segundo Carlomagno ${ }^{42}$, encaja únicamente en un perfil político inclinado hacia posturas de abierta simpatía hacia la entente formada por Francia y Aviñón. Eiximenis

35 ... unde sicut Regnum graecorum, Regnum ispanorum (fol. 37, loc. cit.).

36 Reeves los califica como genuine joaquites (loc. cit., p. 224).

37 Obra conocida a través de los 42 artículos extraídos de su seno por la Inquisición, artículos descubiertos por J.M. Pou y Martí (cf. Visionarios, op. cit., pp. 255-8).

38 Martí de Barcelona ha negado la autoría de esta obra a Eiximenis (cf. "Fra Fr. Eximeniç», Estudis Franciscans, 1928). Sin embargo, la defienden Massó, Pou y Martí y Atanasio López.

39 Cf. M. REEVES, op. cit., p. 222.

40 Desde el Infante Martín al propio Pedro III (cf. M. J. PELÁEZ, «Francisco de Eiximenis y la sociedad eclesiástica de su tiempo", Estudis Franciscanis, 1977, p. 459).

41 Cf. P. BOHIGAS, Profecies catalanes, art. cit., p. 16. Este hecho se conoce a través de una carta del Rey Juan I de Aragón a Pere d'Artés de 17 de Noviembre de 1391. La fecha dada por Eiximenis para el inicio del milenio coincide con la de Rocatallada.

42 Vid. M. REEVES, op. cit., capítulo III: The Second Charlemagne, pp. 320-331. De hecho, Eiximenis incorporó esta profecía hierocrática de la venida de un emperador de los Ultimos Tiempos, subordinado al Papa angélico, que conquistaría Jerusalén. 
se vio pronto obligado a presentar sus excusas al Rey ${ }^{43}$ y denunciaría posteriormente en su Regiment de Princeps como fantasies a las profecías que circulaban sobre el final de los tiempos ${ }^{44}$.

Además, está la cuestión de la imposible conciliación entre el apoyo dado en el $D e$ Triplici Statu Mundi a la posición del papa Urbano VI (que era la encarnación de lo anti-francés en ese momento) y la profecía francófila antes mencionada. Estas extrañas piruetas dadas por el franciscano catalán tienen díficil explicación.

Explicación aún más díficil si atendemos al hecho de que en otra de sus obras, el Primer del Crestiá, Eiximenis se destaca como un abanderado del imperialismo escatológico aragonés. En este tratado afirma sin ambages que ha sido profetizado del Casal aragonés que llegará a conseguir la Monarquía Universal ${ }^{45}$, pues Dios lo habría elegido para grandes tareas, entre ellas la de culminar la Cruzada que destruirá toda la secta de Mahoma ${ }^{4}$.

Esta obra imbuida de la tradición escatológica más genuinamente imperial ha llevado a algún autor a calificar a Eiximenis como a un incondicional de la Monarquía aragonesa, en el cual el sentimiento imperialista catalán arriba a su máximo esplen$d o r^{47}$. Pero, ¿cómo conciliar entonces estas afirmaciones con el mensaje del De Triplici Statu Mund?

Quizá la respuesta la haya ofrecido Ángel López-Amo cuando atribuye a rasgos de indecisión en lo doctrinal y a una falta de voluntad para llevar a su conducta las convicciones de su mente los vaivenes políticos del gran místico franciscano ${ }^{48}$. En efecto, fue en el reino de Aragón donde primero se condenó abiertamente como hereje a Joaquín de Fiore (año 1342) ${ }^{49}$ y era ciertamente aventurado para Eiximenis mostrar inclinaciones de este tipo.

Menor ambigüedad ofrecen los planteamientos de Juan de Rocatallada, radicalmente inclinados hacia las posiciones de Francia y el Pontificado. El fraile de Aurillac, apasionado joaquinita y seguidor de las doctrinas del enigmático visionario fraticelo Pierre Jean d'Olivi ${ }^{50}$, fue uno de los más prolíficos autores proféticos de la Edad Media, destacando entre sus obras el Liber Secretorum Eventuum y el Vade mecum in Tribulatione.

43 El 12 de Diciembre de ese mismo año (cf. P. BOHIGAS, loc. cit., p. 16).

44 Cf. Regiment, cap. 473; vid. P. BOHIGAS, Profecies catalanes, art. cit., p. 17.

45 D'aquesta Casa ès profetat que deu aconseguir Monarchia quasi sobre tot lo Món (Primer del Crestià, Va 247; ed. J. Torras i Bages, La tradició catalana, Barcelona, 1924, p. 323).

46 Car és Casa que Déu ha elegida a fort grandes obres a fer, e a fort meravelloses (Primer del Crestià, op. cit., ed. cit., Va 247).

47 Cf. M. J. PELÁEZ, “Los conceptos França y Espanya en el pensamiento literario y jurídicopolítico de Francesc de Eiximenis», Bulletin Hispanique, 82, 1980, p. 355.

48 Cf. «El pensamiento político de Eximeniç», A. H. D. E., XXIII, 1946, p. 46.

49 Ese año el obispo de Mallorca incluía al abad en su Summa de Haeresibus. Hasta ese momento sólo habían sido condenadas algunas ideas del visionario (el trinitarismo en el concilio de Letrán de 1215, por ejemplo) pero nunca su persona; Reeves, op. cit., p. 69.

50 Cf. M. REEVES, The influence of Prophecy, op. cit., p. 225. 
A pesar de haber sufrido doce años de prisión a manos de la Inquisición Rocatallada se nos aparece como un ferviente devoto de la Iglesia romana. Así, no duda en sus Visiones de 1345 en atribuir a la dinastía aragonesa la continuidad de la simiente venenosa y diabólica de Federico II, afirmando que Pedro III el Grande fue el segundo Anticristo y Federico III de Sicilia el cuarto, de quien nacería el Antichristus magnus ${ }^{51}$. Por contra, en su Vade mecum anuncia que el Rey de Francia será elegido emperador y someterá el totus orbis como fiel ejecutor de las ordenes del Papa angélico ${ }^{52}$.

En estas obras Juan de Rocatallada se hace eco de las ideas del joaquinismo francés primigenio, en particular del Liber de Flore, donde aparece por vez primera la idea de la colaboración con el Papa angélico de un Rex generosus de la línea de los Capeto. Sin embargo, en palabras de Reeves, fue Rocatallada el primero en convertir esta imagen en un completo programa de joaquinismo político al servicio de la Monarquía francesa ${ }^{53}$.

Del mayor interés es su comentario al opúsculo pseudojoaquinita conocido como Oraculum Cyrilli. En él realiza una acabada caracterización de la Casa de Aragón como la dinastía del Escorpión, instrumento del Anticristo y fuente de todos los males políticos del siglo por su condición de sucesores de los Staufen, esa raza maldita, herética y luciférica ${ }^{54}$. Aparecía así como un hecho evidente para Rocatallada la conexión política staúfico-aragonesa. Esta percepción, aunque sea por parte de un enemigo de Aragón, no deja de ser por ello indicativa de la existencia de una opinión extendida al respecto.

\section{PRESENCIA DE FIGURAS ALEGÓRICAS STAÚfICAS EN LA ESCATOLOGÍA IMPERIAL ARAGONESA; POSIBLES LÍNEAS DE CONTINUIDAD SIMBÓLICA E IDEOLÓGICA}

Ha sido Alain Milhou el primer investigador en conceder la importancia debida a la trascendental figura alegórica del Vespertilio en el marco del ciclo profético catalán ${ }^{55}$. Desgraciadamente, no profundizó por completo en las implicaciones que se derivan de su instrumentación como símbolo.

No obstante, ha dejado apuntada la principal vía de explicación del asunto al señalar que, junto a la influencia joaquinita, el imperialismo catalán encontró su justificación en las profecías escatológicas aplicadas a Federico II, profecías que habrían Ilegado al reino de Aragón a través del matrimonio de Constanza de Sicilia con Pedro III,

51 Cf. P. BOHIGAS, Profecies catalanes, art. cit., p. 9.

52 Cf. E. JACOB, «John of Roquetaillade», Bulletin of John Rylands Library, XXXIX, 1956-7, pp. 75-96 y P. Bohigas, art. cit., p. 10.

53 Cf. M. REEVES, op. cit., p. 321. Este programa no era sino la translación escatológica del pensamiento político de Pierre Dubois, quien proclamó la misión cósmica encomendada por Dios al Rey Cristianísimo en su De Recuperatione Terre Sancte.

54 Cf. J. BIGNAMI-ODIER, Etudes sur Jean de Roquetaillade, París, 1952, pp. 53-109.

55 Cf. La Chauve-Soris, le Nouveau David et le Roi Cache, art. cit. 
un enlace que convirtió al monarca aragonés en el heredero de las ambiciones gibelinas universales de la Casa imperial de Suabia ${ }^{56}$.

Los síntomas de la formación de una incipiente escatología, reflejo de la toma de conciencia imperial catalana, abundan en el último cuarto del siglo XIII. La Crònica de Ramón Muntaner ${ }^{57}$ o el Llibre dels Feits de Jaime I son buenos ejemplos de ello. En este sentido, la obra autobiográfica del Rey Conquistador nos ofrece el primer testimonio del ciclo profético imperial: la visió del menoret. El sueño profético de un franciscano (como no podía ser de otro modo) de Navarra anunciaba al Rey Jaime que un Rei d'Aragó que ha nom Jacme habría de restaurar tots los crestians en Espanya $a^{58}$.

A estos primeros indicios de una escatología autóctona hay que sumar el papel decisivo que sin duda jugó la transmisión de la mentalidad staúfica a través de los exiliados de Sicilia que llegaron a Aragón con Constanza ${ }^{59}$.

La recepción de la mística escatológica staúfica en la corte aragonesa se centró básicamente en dos figuras alegóricas: la imagen hespérica y la efigie del Dragón como símbolo mesiánico de la Realeza.

No exageramos al afirmar que la imagen hespérica fue la plasmación literaria más acabada, en un lenguaje mítico-simbólico, de esa translatio Imperii que, de alguna forma, se produjo entre los Staufen sicilianos y los hispánicos (entendiendo por estos últimos a ambas ramas, la castellana y la aragonesa, nacidas de los matrimonios de Beatriz de Suabia y Constanza de Sicilia con sendos monarcas ibéricos) ${ }^{60}$.

Tras la muerte del Rey Manfredo en la batalla de Benevento (1266), esta translatio apareció como algo evidente para algunos círculos gibelinos italianos y

56 Limperialisme catalan, qui s'était rendu maître de la Sicile dès 1282, pouvait trouver sa justification dans les prophéties eschatologiques jadis appliquées à l'empereur Frédéric II de Hohenstaufen, Roi de Sicile et de Jérusalem; en effect, per le mariage de Pierre III avec Constance, le petite fille de Fréderic, les rois d'Aragon avaient hérité des ambitions gibellines universelles de celuici... (A. MILHOU, art. cit., p. 62).

57 Muntaner hace exclamar al Papa: Que cascun cavaller d'aquells de Catalunya són diables encarnats... aquestes són gents ab qui conquistariem lo Món, e metria'n hom abaix tots los infeels (cf. Crònica, c. CXLIX, p. 7, t. II, ed. M. Gustà, Barcelona, 1979).

58 Cf. Llibre dels Feits, c. CCCLXXXIX, p. 326, ed. Ferrán Soldevilla, Barcelona, 1982; esta profecía era aplicable también a Jaime II ¿fruto de una interpolación posterior?

59 Helene Wieruszowski considera di grande importanza el papel jugado en la corte aragonesa por estos exiliados, destacando figuras como las de Juan de Prócida, Roger de Lauria, Conrado y Manfredo Lancia, Francisco Trogisio, Nicolás de Aspello... ("La corte di Pietro d’Aragona", en Politics and Culture in Spain and Italy, Roma, 1971, p. 201).

$60 \mathrm{El}$ fecho del /mperio en que se vio envuelto Alfonso $X$ no es un fenómeno que se pueda desligar del Vespro siciliano y los inicios del imperialismo aragonés. De hecho, Pedro III no hizo sino recoger el testigo en Italia del liderazgo gibelino ejercido allí por el Rey de Castilla entre 1268 y 1280. Por otro lado, el Rey Sabio fue objeto de una campaña similar a la sufrida posteriormente por Aragón: un joaquinita tan destacado como Gerardo del Borgo San Domnino (autor de la Introducción al Evangelio Eterno) le tildó de ser el Anticristo en 1258 (vid. Antonio BALLESTEROS, Alfonso X, Barcelona, 1984). 
alemanes $^{61}$. Apenas dos años después circulaba en Alemania e Italia la profecía Regnabit Menfridus que anunciaba el advenimiento de un Tertius Fridericus, nomine orientalis, que destruiría al León francés (cuya encarnación era Carlos de Anjou) ${ }^{62}$. Aunque el Federico del Oriente al que se alude en la profecía era un alemán, el hijo del landgrave de Turingia ${ }^{63}$, se vaticinaba en ella que la destrucción de Francia sólo se produciría tras una confederación de Alemania con los reinos españoles: Theutonici et Hyspani confederabuntur et Regnum Francie redigent in nichilum ${ }^{64}$.

Una docena de años después un canónigo gibelino de Colonia, Alejandro de Roes (célebre por sus obras políticas en defensa de un Imperio Universal germánico) al poco tiempo de haber dado continuidad a la profecía del Tercer Federico en su De translatione Imperii $(1281)^{65}$, componía un extraño epigrama satírico, el Prologus in Pavonem (1285).

En este breve opúsculo Alejandro de Roes realiza una alegoría animal del Concilio de Lyon (1245), en la que el Papa Inocencio IV, simbolizado por un Pavo, arrebata injustamente el Imperium sobre los animales al Aquila (el emperador Federico II) con el fin de entregárselo al intrigante Gallus (el Rey de Francia). El final de la sátira consiste en una profecía en la que Alejandro de Roes, un enemigo declarado del papa Martín IV y de Francia, anuncia que la injusticia cometida con el Aquila será vengada por los falcones peregrinos (los españoles) ${ }^{66}$.

Ahora bien, este anuncio, aunque tiene de por sí grandes implicaciones, sólo cobra toda su significación a la luz del enigmático último verso del poema: quos illis Grecus, Calaber transmisit et Hesper ${ }^{67}$. El editor, Herbert Grundmann, interpretó esta alusión

61 Ello es fácilmente comprobable asomándose a las crónicas gibelinas del norte de Italia compuestas en esos años. Los Anales lanuenses, los Anales de Oberto Stanconi, los Anales lacobi Aurie o los Anales Placentini Gibellini confirman esta impresión (publ. en los M. G. H., Annales Italiae, $t$. XVIII, Hannover, 1863, pp. 228-574).

62 Cf. Chronica Minor Auctore Minorita Erphordiensi, M. G. H., XXIV, p. 207. Esta profecía, originada en un ámbito gibelino italiano, fue llevada a Alemania por el cardenal-obispo de Porto (cf. M. REEVES, The influence of Prophecy, op. cit., p. 313). De similar contenido era la profecía antifrancesa Gallorum levitas, gestada en esos años.

63 Cuya madre era una Staufen; cf. M. BLOOMFIELD y M. REEVES, «The Penetration of Joachism into Northern Europe», Speculum, 29, 1954, p. 790.

64 Cf. Chronica Minor, loc. cit.; Esta profecía no hacía sino recoger las esperanzas depositadas por los gibelinos italianos en Castilla y Aragón, las únicas potencias europeas que tras la batalla de Tagliacozzo desafiaban aún al bloque güelfo-angevino.

65 De huius Frederici germine radix peccatrix erumpet Frederico nomine qui clerum in Germania et etiam Romanam Ecclesiam valde humiliabit et tribulabit vehementer (ed. H. Grundmann, «Die schriften des Alexander von Roes", Kritischen Studientexte der M. G. H., IV, Weimar, 1949, p. 30).

66 Ut qui nolebant Aquila regnante modeste,

conregnare sibi contenti finibus illis,

Quos natura deditm discant moriendo rebelles

Bubones, milvos et falcones peregrinos... (ed. H. Grundmann, Die schriften des Alexander von Roes, op. cit., vv. 268-271, p. 122). La identificación de los falcones con los hispani es realizada por el propio Alejandro de Roes es el proemio al poema.

67 Prologus in Pavonem, v. 272, p. 122, ed. cit. 
al Hesper como un anuncio, concebido abiertamente como un vaticinium ex eventu, de las Vísperas sicilianas ${ }^{68}$.

Era ésta la primera vez que se realizaba el fácil juego de palabras alegórico entre el acontecimiento del Vespro y el nombre que la mitología clásica había otorgado a la península ibérica. No obstante, la elección de la noción Hesperia como figura alegórica no se debió únicamente a una similitud semántica y formal con el término latino para la tarde, sino al conjunto de connotaciones que tenía en la literatura pagana clásica como sinónimo del Occidente más extremo, del Poniente ignoto sede del Jardín de las Hespérides y de los Campos Elíseos.

En efecto, la publicística staúfica ya había utilizado la imagen hespérica en su escatología más primigenia. El médico de la corte siciliana, Pedro de Eboli, al glosar el nacimiento de Federico II en su De rebus Siculis carmen (1195) y profetizar los triunfos que alcanzaría, menciona que su nativitas se había producido ab Experia ${ }^{69} \mathrm{y}$ califica a su madre, la reina Constanza, como Experiam diem, la luz del Occidente (v. 1063). Y, dentro del ciclo escatológico imperial bizantino (que tanta influencia ejerció en el staúfico), se anunciaba desde el siglo XII la conquista de Jerusalén por parte de un Rey de cabellos rubios, al que se denomina Estrella del Poniente ${ }^{70}$.

Alain Milhou menciona a este respecto una profecía castellana de finales del siglo XIII atribuida al sabio árabe Acham Turuley que anuncia la venida de un Rey mesiánico en los últimos fines de Poniente ${ }^{71}$. Siendo España el país más occidental de la Cristiandad era lógico que la imagen hespérica se convirtiera pronto en un motivo recurrente para el ciclo profético catalán.

De esta forma, la ya mencionada Refundició denomina de forma reiterada Rei Pere de Ponent a Pedro III, aludiendo a un misteri de Ponent iniciado por el monarca aragonés al conquistar Sicilia y a culminar con el sant passage a Jerusalén ${ }^{72}$. El nomen orientalis del Tercer Federico alemán iba a dejar así paso al misteri de Ponent aragonés.

La imagen del Vespertilio de Arnaldo de Vilanova tuvo la virtud de aúnar gráficamente en un único símbolo esta idea del monarca mesiánico venido de Occidente con la figura staúfica del Dragón. De ahí su posterior difusión.

Al igual que el Aguila era comúnmente interpretada como símbolo del Imperio romano en general y de Alemania en particular, el Dragón (cuyas connotaciones eran

68 En la introducción a su edición de las obras de Alejandro de Roes, Grundmann señala que los versos 270-272 son un prophetisch anspielen del Sizialianische Vesper (cf. Die schriften des Alexander von Roes, op. cit., p. 12). Por otro lado, en su traducción del poema intercambia claramente el término Hesper por Spanien.

69 Venit ab Experia nativi paima triumphi (cf. «De rebus Siculis carmen», ed. L.A. Muratori, v. 1363, Rerum Italicarum Scriptores, XXXI, 1094, p. 177). Muratori indica que el término Experia o Hesper se aplica por igual a Italia o España, tierras occidentales.

70 Cf. Paul ALPHANDÉRY, La Chretiente et lidée de Croisade, París, 1954, II, p. 91.

71 Cf. ms. de la B. N. de Madrid, $n^{\circ}$ 18725; A. MILHOU, Le Chauve Soris, art. cit.

72 Cf. Refunfició compendiada de la Summa Concordiae Veteris et Novi Testamenti, op. cit, apud Pere Bohigas, Profecies catalanes, art. cit., pp. 8-9. 
claramente diabólicas para la escatología de la Iglesia), se había convertido de alguna forma en el emblema de los Staufen. Así, el ciclo legendario gibelino narraba que la reina Constanza, había soñado ${ }^{73}$ poco antes de dar a luz que de su vientre iba a surgir un Dragón cuyo fuego abrasaría toda Italia. El apelativo filius Draconis sería repetidamente aplicado en el futuro a Federico II tanto por parte de sus enemigos ${ }^{74}$ como por sus fieles. $Y$ es que en la mentalidad mitológica germánica el Dragón no tenía las mismas connotaciones negativas que en la judeo-cristiana. Wolfram von Eschenbach, por ejemplo, relata que la madre de Parsifal, Herzeloïde, había soñado también que iba a dar a luz a un Dragón ${ }^{75}$.

El infante aragonés don Pedro, miembro de la Orden franciscana, recogió en el año 1347 la imagen vilanoviana del Vespertilio y la insertó en la estructura de la Prophetia Tripolitana, una visión tenida por un monje cisterciense en Tierra Santa, cuya primera versión circuló hacia $1240^{76}$.

El emperador de los últimos tiempos conquistador de Jerusalén anunciado por el monje libanés se convierte en la versión latina de don Pedro en el Vespertilio ${ }^{77}$. En su glosa en catalán el Infante minorita va aún más lejos y vaticina que la Rata pinyada (el Vespertilio) destroira les mosques d'Espanya e sotsmetra a si Affrica e pendra la senyoria general del Mon, ço es l'Imperi ${ }^{8}$. El programa imperial aragonés era así recogido en su integridad por quien, reuniendo en su persona la doble condición de infante y franciscano, era el más indicado para formularlo en clave escatológica.

Dos secuelas tardías de la imagen del Vespertilio se encuentran en el Primer del Crestiá de Francisco de Eiximenis, donde aparece una difusa alusión a la Rata pennada ${ }^{79}$, y en la Explicació d'alguns secrets de l'Escriptura (año 1449), que hace del Rey aragonés Alfonso IV la última encarnación del Vespertilio ${ }^{80}$, del que dice ser el fill de l'Aguila ¿Tendría conciencia el anónimo autor del origen alemán del Murciélago aragonés?

73 Cf. Ernst H. KANTOROWICZ, Kaiser Friedrich der Zweite, op. cit., c. I.

74 Una profecía joaquinita de 1264 llama a Federico II Draco repletus furore; Para Reeves es un testimonio de la popularidad del Dragon-symbol (cf. The influence of Prophecy, op. cit., p. 51).

75 Aspecto apuntado por KANTOROWICZ; loc. cit.

76 Esta profecía conoció una larga y exitosa serie de reediciones en 1240, 1287, 1289, 1291 y 1297 siendo esta última la que glosa el Infante argonés; cf. Robert E. LERNER, «Medieval Prophecy and religious dissent”, Past and Present, 72, 1976, pp. 12-13.

77 ... cedrus alta Libani succidentur et Tripolis in brevi destruetur et aconcapietur et maris Saturnus insidiabitur Jovi et Vespertilio fugavit eum (apud P. BOHIGAS, Profecies catalanes, art. cit., p. 11, n. 3).

78 Ms. de la B. Mun. de Carpentras, n 336, f. 76r, apud P. BOHIGAS, art. cit., p. 11.

79 Cf. POU Y MARTÍ, Visionarios, op. cit., pp. 397-415; Milhou, La Chauve Soris, art. cit.; esta alusión de Eiximenis indica su conocimiento del significado de la alegoría, pero no que compartiera el mensaje ímplicito en ella.

80 Cf. P. BOHIGAS, art. cit., p. 24. 